\title{
La combativité cheminote en question : la grève avortée de novembre 1974
}

Railwaymen's combativeness in question: the missed strike of November, 1974

\section{Alix Ducamp et Vincent Bouget}

\section{OpenEdition}

\section{Journals}

Édition électronique

URL : https://journals.openedition.org/rhcf/159

DOI : $10.4000 /$ rhcf.159

\section{Éditeur}

Rails \& histoire

\section{Édition imprimée}

Date de publication : 1 décembre 2007

Pagination : 308-328

ISSN : 0996-9403

Référence électronique

Alix Ducamp et Vincent Bouget, «La combativité cheminote en question : la grève avortée de novembre $1974 »$, Revue d'histoire des chemins de fer [En ligne], 36-37 | 2007, mis en ligne le 10 mai 2011, consulté le 22 avril 2022. URL : http://journals.openedition.org/rhcf/159 ; DOl : https://doi.org/ 10.4000/rhcf.159 


\title{
La combativité cheminote en question : la grève avortée de novembre 1974
}

\author{
Alix Ducamp \\ professeur d'histoire-géographie, académie de Créteil, maître en \\ histoire, université de Paris I - Panthéon-Sorbonne \\ Vincent Bouget \\ professeur d'histoire-géographie à Sarcelles (Val d'Oise), maître \\ en histoire, université de Paris I - Panthéon-Sorbonne
}

Depuis les années 1920, les cheminots bénéficient d'une réputation de combativité dans l'imaginaire collectif de la société française. Cette réputation est étayée par de grands moments de l'histoire du mouvement ouvrier et elle est portée par l'influence d'acteurs politiques et syndicaux importants dans la France des Trente Glorieuses : la CGT et le PCF qui ont, à l'époque qui nous concerne, une forte assise à la $\mathrm{SNCF}^{1}$. Leur réputation est pourtant entamée par des échecs ou semiéchecs, notamment en 1971 où, après un mois de grève ou presque, les cheminots n'obtiennent presque rien ${ }^{2}$.

En novembre 1974, les cheminots se trouvent dans une situation particulière. Les postiers ont engagé une grève illimitée et générale depuis octobre. La conjoncture, notamment le gouvernement Giscard récent, laisse penser qu'ils ont tout intérêt à élargir leur mouvement, naturellement en premier lieu en direction des cheminots, et ce, pour plusieurs raisons. D'abord, ils partagent leurs lieux de travail : les gares sont aussi des centres de tri. Ensuite, les cheminots ont précisément cette réputation de combativité : ils ont bien lutté côte à côte en 1953. Enfin, leurs revendications au sein de la fonction publique se rejoignent, tant sur la question des salaires qu'à propos des conditions de travail et de statut, ainsi que sur la question de l'auxiliariat.

Un mot d'ordre de grève circule effectivement à la SNCF début novembre 1974 : il est axé sur des revendications catégorielles mais on suppose que la jonction avec les postiers peut avoir lieu. Or le mouvement ne "prend" pas : après une série de grèves désordonnées, décidées

\footnotetext{
1- Par exemple, selon un rapport des Renseignements généraux daté du 10 février 1973, il y a $40 \%$ de syndiqués parmi les cheminots, la CGT étant majoritaire.

2- Voir Christian Chevandier, Cheminots en grève ou la construction d'une identité: 18482001, Paris, Maisonneuve \& Larose, 2002, 399 p.
} 
localement sans mot d'ordre national, le mouvement s'éteint doucement à la SNCF avec le week-end prolongé du 11 novembre, pour reprendre du 18 au 22 novembre, sur le mode des grèves tournantes, le 19 marquant le point culminant de la mobilisation à l'occasion d'une grande journée nationale et interprofessionnelle d'action. Peu de perturbations sont signalées dans la circulation des trains d'autant plus que FO et la Fédération générale autonome des agents de conduite refusent de prendre part au mouvement.

Il s'agit donc pour nous de comprendre l'image des cheminots qui s'est formée dans la société (au sens des acteurs syndicaux, médiatiques, politiques - en particulier l'extrême gauche -, des cheminots et postiers eux-mêmes) pendant et à l'issue de la grève des postiers en novembre 1974. Autrement dit : la grève des postiers et le mouvement raté qui s'en est suivi à la SNCF ont-ils eu des répercussions sur les représentations de la combativité cheminote? Comment caractériser les attentes qui se fixent sur les cheminots pendant la grève des postiers ? Et quelles sont les réactions à l'échec d'un mouvement cheminot en novembre 1974 ?

\section{Les attentes suscitées par les cheminots...}

Dès le départ, il semble exister une césure entre les attentes des acteurs internes (c'est-à-dire les syndicats ${ }^{3}$, les Renseignements généraux liés à la direction de la $\mathrm{SNCF}^{4}$ et bien sûr à l'État, les travailleurs cheminots et postiers - eux-mêmes) et les acteurs externes (médias : presse et télévision ${ }^{5}$, les différents acteurs politiques en particulier l'extrême gauche $^{6}$, très peu implantée ${ }^{7}$ à la SNCF), ce qui est somme toute assez traditionnel mais qui prend ici une forme particulière : ce sont en effet les acteurs externes qui présentent le plus d'attentes vis-à-vis des cheminots.

3- Nous avons dépouillé les archives syndicales suivantes : fédérations CGT des PTT et des cheminots, fédérations CFDT des PTT et des cheminots ainsi que les Archives confédérales de la CFDT.

4- Archives de la préfecture de police (désormais : APP), carton GA S30, chemise « SNCF. Informations générales 1974 ».

5- Médias consultés: Le Parisien, L'Humanité, Le Monde, Le Figaro, Minute, L'Express ainsi que les notices descriptives des journaux télévisés des trois chaines de télévision.

6- De nombreux tracts et journaux ont pu être consultés à la BDIC, ainsi que quelques tracts du PCF, du PSU et du PS.

7- «L'implantation », dans les organisations politiques, en particulier d'extrême gauche, consiste à installer de façon plus ou moins durable des militants dans un lieu donné pour faire la propagande de l'organisation et pour recruter de nouveaux militants. 
... chez les postiers, les syndicats et les Renseignements généraux

En effet, les acteurs internes, présents sur le terrain, semblent plus circonspects dans leurs attentes envers les cheminots. D’après plusieurs témoignages et d'après les syndicats majoritaires chez les postiers (CFDT, CGT, FO), il n'y a pas eu de la part des postiers d'appels clairs à l'attention des cheminots. Par exemple, un ancien postier des PLM s'étonne de cette idée : «On était dans notre mouvement. Non, on n’a pas compté sur les cheminots ${ }^{8}$. $\gg$ Parmi les nombreux tracts conservés par la fédération cégétiste des PTT, on ne trouve aucune demande de soutien destinée aux cheminots, mais seulement des appels de solidarité lancés aux usagers ou au mieux à l'ensemble de la fonction publique, les cheminots ne se distinguant pas particulièrement. Dans ses communiqués internes envoyés aux syndicats locaux, la fédération CFDT-PTT rappelle la nécessité d'être en lien avec les autres salariés en grève dans la fonction publique comme dans le secteur public. Si les cheminots sont cités, et même lorsque ceux-ci sont dans l'action', ils ne sont qu'un secteur parmi d'autres. Seules les organisations d'extrême gauche dans des tracts et bulletins de boîte encouragent clairement les postiers à faire appel aux autres salariés de la fonction publique : « Aujourd'hui la question de l'unité avec les camarades du secteur public et nationalisé devient décisive. Vous pourrez la résoudre. [...] C'est en vous rendant directement auprès des cheminots que vous pourrez réaliser l'unité avec eux ${ }^{10}$. »

De la part des cheminots eux-mêmes, il semble que le même constat s'impose : d'après quelques indices, les cheminots n'ont pas voulu se lancer dans un mouvement de solidarité avec les postiers. On le voit d'abord d'après les collectes mentionnées dans des tracts des archives de la fédération CGT des PTT : les cheminots ont relativement peu donné pour la grève des postiers, en comparaison avec d'autres professions, de la fonction publique ou même du secteur privé. Surtout, selon les rapports des Renseignements généraux, la participation des cheminots à la grève a été assez faible. Enfin, à la CFDT, on précise bien, à l'époque, qu'un mouvement de solidarité doit être un mouvement de grève sur ses propres revendications et si possible sur des revendications communes, mais qu'il n'est pas souhaitable de faire grève simplement pour soutenir d'autres corporations. Cependant des tracts et des journaux

8- Nous avons pu recueillir quelques témoignages d'anciens postiers et d'anciens cheminots syndicalistes.

9- Archives fédérales CFDT-PTT, Communiqué interne $n^{\circ} 32$ du 9 novembre et $\mathbf{n}^{\circ} 33$ du 10 novembre.

10- Tract du parti communiste révolutionnaire-front rouge, archives de la BDIC. 
d'extrême gauche évoquent par moments une mobilisation importante et enthousiaste des cheminots au début de la grève, ou mentionnent des élans de solidarité entre les deux catégories de travailleurs. Par exemple, selon Lutte ouvrière, des cheminots ont empêché des CRS d'évacuer des grévistes postiers d'une gare ${ }^{11}$. De même, selon un journal anarchiste, le Rail enchainé, vraisemblablement rédigé par des cheminots libertaires, les gares et dépôts étaient prêts à faire grève : "L'impression qui se dégage, c'est que les cheminots ont envie d'y aller [...] des cheminots demandent de durcir la grève [lors d'une assemblée générale] [...] Vendredi 8 novembre. Nous avons défilé sur les mots d'ordres suivants : "la gare, la poste, l'usine aux travailleurs : une solution, la grève générale comme les postiers, grève illimitée [...] cheminots-postiers : même patron." $»^{12}$ Il est difficile de mesurer s'il s'agit d'un activisme cheminot isolé ou d'un phénomène dont ni les renseignements généraux, ni les syndicats, ni les médias ne rendent compte. Dans tous les cas, la mobilisation a pu être forte par endroits, mais reste faible en règle générale.

Ce qui peut apparaitre comme un manque de solidarité de la part des cheminots en direction des postiers a d'ailleurs plusieurs explications. Beaucoup de syndicalistes de la CGT, dont Georges Lanoue et Jean-Michel Sgorlon ${ }^{13}$, avancent l'impact négatif de la grève de 1971. Par ailleurs, les Renseignements généraux (RG), dans un premier temps, peuvent croire à la naissance d'un mouvement de masse cheminotpostier car le climat social est tendu ${ }^{14}$. Mais ils constatent rapidement que la situation se calme, puisque la direction de la SNCF, alors que le mot d'ordre de grève est dans l'air, accorde $4 \%$ d'augmentation sur les salaires, ce qui est considérable ${ }^{15}(1 \%$ d'augmentation seulement avait été consenti par l'accord salarial de 1974). D'après les RG, la direction de la SNCF a pris toutes les dispositions pour que la grève échoue, pour « résorber le mouvement», selon l'expression utilisée dans un rapport.

11- Lutte ouvrière, $\mathrm{n}^{\circ} 325$, daté du 19-25 novembre 1974, p. 6.

12- Le Rail enchainé, $\mathrm{n}^{\circ}$ 5, consulté à la BDIC dans un dossier Cahiers de Mai, SNCF.

13- Jean-Michel Sgorlon, actuel secrétaire général adjoint de la fédération CGT des cheminots, jeune cheminot dans les années 1970 et Georges Lanoue, ancien secrétaire général de la fédération à partir de la seconde moitié des années 1970, que nous avons pu rencontrer.

14- APP, Rapports du $1^{\text {er }}$ octobre 1974 et du 11 octobre 1974.

15- Ibid., le rapport du $1^{\text {er }}$ novembre 1974 s'intitule : «L'attribution d'une augmentation de $4 \%$ aux cheminots semble désamorcer les actions locales en préparation. » 
$\mathrm{Du}$ reste, les syndicats sont particulièrement prudents. FO, engagée dans la participation avec la direction de la SNCF la fédération a signé l'accord salarial de 1974, contrairement à la CGT, la CFDT, la CFTC et la FGAAC) s'oppose rapidement à ce mouvement. Dans un communiqué fait à la suite d'une réunion avec la direction de la SNCF, cité par les Renseignements généraux ${ }^{16}$, $\mathrm{FO}$ vante « le bien-fondé de la politique de concertation » qu'elle mène. Dans la suite du rapport, les Renseignements généraux estiment que la position de force de $\mathrm{FO}$ (la direction de la SNCF vient d'annoncer les $4 \%$ d'augmentation négociés avec les organisations signataires de l'accord salarial de 1974, principalement FO) va influer sur le conflit en préparation et « désamorcer les velléités d'action dans les secteurs où l'implantation de [ce] syndicat est efficace ». Le syndicat s'affiche aussi systématiquement dans les médias contre la politisation des grèves que représente pour lui la généralisation du mouvement des postiers à l'ensemble de la fonction publique. Part ailleurs, la Fédération générale autonome des agents de conduite, essentielle pour une grève véritablement efficace à la SNCF puisque c'était à l'époque le syndicat majoritaire des roulants, reste sur la réserve parce que les revendications ne tiennent selon elle pas assez compte des catégories. Les Renseignements généraux sont là encore une source essentielle, puisqu'ils précisent dans leurs rapports les tensions qui règnent entre CGT et FGAAC, qui les amènent à conclure à un apaisement du mouvement en préparation. Quant à la CGT, elle se contente d'appeler prudemment à des actions locales et donc à une gestion locale du mouvement, alors que la CFDT est l'organisation qui se montre la moins frileuse, selon les RG et selon des organisations trotskistes comme l'Alliance marxiste révolutionnaire : elle est la seule à pousser véritablement à l'action, tout en étant freinée par sa coopération et par son attentisme vis-à-vis de la CGT. Les Renseignements généraux précisent par exemple que la CGT veut attendre la fin des vacances de la Toussaint pour lancer une action alors que la CFDT souhaitait partir beaucoup plus $v_{i t e}{ }^{17}$. Là encore, les Renseignements généraux rapportent cette division et cette inaction syndicales, qui pour eux condamnent sans appel le mouvement: «Après le refus des dirigeants de la SNCF et du secrétariat d'État aux Transports de recevoir les responsables CGT, CFDT et CFTC, le conflit actuel devrait tendre vers un pourrissement se traduisant par une reprise normale du trafic ferroviaire à partir de la semaine prochaine. Le conflit en cours à la SNCF entre dans sa phase finale ${ }^{18}$. »

16- Ibid., Rapport du $1^{\text {er }}$ novembre 1974.

17- Ibid., Rapport du 4 novembre 1974.

18- Ibid., Rapport du 7 novembre 1974. 
Quelques jours auparavant, un autre rapport soulignait l'inertie des syndicats en l'expliquant par leur indécision : si «la tension qui règne actuellement aux PTT et dans une moindre mesure chez certaines catégories d'agents de la SNCF est ressentie par les responsables syndicaux des cheminots CGT, CFDT, CFTC », néanmoins il semble aux Renseignements généraux que ces responsables « aient des difficultés à "sentir la base" qui présentement ne parait pas très mobilisée. Aussi leurs efforts consistent-ils à susciter des réunions, autant pour s'informer de l'état d'esprit des militants que pour tenter de les mobiliser $\rangle^{19}$. Toutefois, les Renseignements généraux précisent que « cet affaiblissement de la combativité des cheminots n'exclut pas leur participation à un conflit national interprofessionnel $»^{20}$ d'une journée. On remarquera que, pour décrire l'attitude des syndicats des cheminots au début du mouvement, notre source principale est les rapports des RG : en effet, les syndicats ont finalement assez peu communiqué publiquement sur cette affaire, à tel point qu'il n'existe d'ailleurs pas de numéro de novembre de la Tribune des cheminots, le journal de la fédération CGT, ce qui est une première (il n'y a pas d'autre numéro manquant dans l'histoire de ce journal après la Seconde Guerre mondiale). Cependant, pour nuancer nos propos, il faut citer cet appel commun des confédérations CGT et CFDT, cité par Vie ourrière, le journal confédéral de la CGT, le 19 novembre : « [la CGT et la CFDT] invitent tous les travailleurs à renforcer leur action et à organiser la riposte et la solidarité de masse autour des travailleurs des PTT en lutte. » De plus, les numéros de « l'édition cheminots » de Syndicalisme-Hebdo sont introuvables pour cette période dans les archives fédérales CFDT. Dans l'édition confédérale de Syndicalisme-Hebdo, les cheminots ne sont pas le seul secteur à être l'objet d'un article. Même s'il est vrai que, dans son édition du 7 novembre, les cheminots se retrouvent en deuxième position derrière les PTT dans les «luttes dans le secteur public », rien ne montre une envie de les mettre particulièrement en valeur. On rappelle leur mécontentement, leur action de grève et leur revendication mais ils restent un secteur parmi d'autres.

Des documents provenant des Archives confédérales de la CFDT semblent offrir une vision des positions syndicales face à la possibilité d'un mouvement cheminot différente de celle des RG. Ainsi, lors d'un comité des fédérations ${ }^{21}$, le 30 octobre, la CFDT ne se montre pas aussi

19- Ibid., Rapport du 4 novembre 1974.

20- Ibid., Rapport du 7 novembre 1974.

21- Archives confédérales de la CFDT, Carton $8 \mathrm{H} 400$, activité du secteur actionrevendication (octobre-décembre 1974). 
optimiste que la CGT à propos d'une grève illimitée. Un relevé de décisions du secteur action-revendication de la CFDT, daté du 31 octobre et faisant suite à une rencontre avec la CGT et la FEN, précise que la « CGT pense que les cheminots vont partir ${ }^{22}$. Cette divergence d'appréciation est rappelée lors du bureau national de la CFDT les 7 et 8 novembre 1974 : « [la CGT] a surestimé les possibilités d'action des travailleurs et notamment la combativité des cheminots. Elle a surestimé également la capacité des militants communistes à mettre en grève les travailleurs des entreprises nationalisées ${ }^{23}$.» La CFDT, sceptique quant à un mouvement d'ampleur à la SNCF, n'exclut toutefois pas, toujours lors du bureau national des 7 et 8 novembre, des actions dans le secteur public et nationalisé dans le cadre d'une journée d'action interprofessionnelle. Ce bureau national se donne précisément pour objectif un « développement de l'action » et la mise en place d'une journée nationale qui «ne doit pas apparaitre comme le relais de l'action des PTT », car « les secteurs ont à se battre pour leurs propres problèmes : c'est cela la solidarité de classe, ce n'est pas une journée de solidarité » ${ }^{24}$. La CFDT, qui est beaucoup moins présente à la SNCF que la CGT, ne semble pas croire à une mobilisation puissante des cheminots. Le 16 novembre, le secteur action-revendication, dans une lettre d'information sur «la situation revendicative et le conflit des PTT », confirme ce qui était analysé dix jours plus tôt et ajoute que c'est la CGT cheminots qui a suspendu cette grève au bout de trois ou quatre jours. Dès lors, même s'il est rappelé que «la combativité existe dans [les secteurs publics et nationalisés] » " pour des luttes importantes », il est « peu probable » qu'un démarrage de ces secteurs dans l'action sur le modèle PTT soit «crédible »" En résumé, des actions importantes sont possibles, mais la combativité des salariés ne permet pas d'envisager un mouvement généralisé et illimité dans la fonction publique, l'échec de la CGT pour faire démarrer les cheminots en est la preuve.

\section{2- Ibid.}

23- Archives confédérales de la CFDT, Carton 8H68, débats au bureau national concernant l'action revendicative de la CFDT, extraits des comptes rendus des sessions (avril 1974-avril 1982).

24- Ibid.

25- Archives confédérales de la CFDT, Carton 8H400, activité du secteur actionrevendication (octobre-décembre 1974). 


\section{... Dans les mouvements d'extrême gauche}

Au contraire, dans l'extrême gauche ${ }^{26}$, les attentes sont grandes : l'élargissement du mouvement des postiers apparait comme une évidence. Sur ce point, les tracts sont nombreux et les organisations unanimes, qu'elles soient trotskistes, anarchistes ou maoistes : selon la Ligue communiste révolutionnaire (LCR), qui s'exprime par un tract: « Postiers-cheminots au coude à coude montrent la voie ! »; selon l'Alliance marxiste révolutionnaire (AMR), dans un bulletin de boite, $L a$ Riposte, daté de la mi-novembre : « Les objectifs du pouvoir sont clairs : miser sur la limitation et la dispersion des grèves dans le secteur public et ailleurs... Plus que jamais il faut élargir la grève des PTT contre le même patron : l'Etat. » L'occasion d'une grève générale est à saisir, selon un article de Rouge, le journal de la LCR : «Si les postiers restent isolés, il sera difficile de retrouver meilleure occasion ${ }^{27}$. » Un autre argument consiste à dire que si l'occasion de la lutte est manquée, les conséquences en seront désastreuses pour tous les travailleurs : «Demain, si nous laissons réussir cette opération [c'est-à-dire le gouvernement brisant la grève des postiers], ce sera difficile de surmonter le découragement, et pas seulement aux PTT, mais dans toutes les branches ${ }^{28}$.» Une seule exception notable, l'Organisation communiste internationaliste (OCI), pour qui le mouvement des postiers doit avant tout rechercher l'unité syndicale. Cette position particulière, surprenante de la part d'une organisation trotskiste, s'explique par les forts liens qui unissent l'OCI à FO : les lambertistes cherchent donc à rejeter la faute du désengagement de FO dans le mouvement des PTT sur la division syndicale et sur l'attitude de la CGT et de la CFDT.

Il apparait clair à l'extrême gauche que les revendications des cheminots et des postiers peuvent et doivent se rejoindre. La Taupe rouge, «bulletin de boite » de la LCR à la SNCF, le proclame à plusieurs reprises : "il faut une plate-forme de la fonction publique »; "Travailleurs des services publics et du secteur nationalisé! Les

26- Nous avons consulté des tracts et des journaux d'organisations trotskistes (Lutte Ouvrière, Ligue communiste révolutionnaire, Organisation communiste internationaliste, Alliance marxiste révolutionnaire), maoistes (parti communiste marxiste-léniniste de France-humanité rouge, Parti communiste révolutionnaire- front rouge, principalement), des publications anarchistes liées à la Confédération nationale du travail comme Le Rail enchainé ou Le Postier affranchi, ainsi que les archives des Cabiers de mai de la BDIC.

27- Rouge, $\mathrm{n}^{\circ} 272$ (1 ${ }^{\text {er }}$ novembre 1974), p. 1-2.

28- « Bulletin de boîte» du PCR-front rouge, Le Postier marxiste-léniniste, non daté, archives de la BDIC. 
revendications des postiers sont nos revendications à tous. » « L'extension de la lutte est possible sur la base de revendications communes », comme l'affirme un numéro du Postier marxiste-léniniste; " Chez les cheminots, le manque d'effectifs est évalué à plusieurs milliers, et plusieurs débrayages locaux ont déjà eu lieu sur le problème. Les salaires ne valent pas mieux que dans les P. et T. » selon un article de Rouge déjà cité. Les cheminots sont ainsi vus comme un allié essentiel, car très combatif, et qui a aussi toutes les raisons de faire grève. C'est un phénomène qui montre la puissance du mythe du cheminot (comme celui du mineur ou du métallo) ${ }^{29}$ dans l'extrême gauche des années 1970. Dans la vision du monde du travail de ces organisations, les cheminots sont une couche sociale plus révolutionnaire que les postiers : par leurs conditions socioéconomiques, leurs conditions de travail et l'histoire de leur corporation, ils appartiennent à l'avant-garde du prolétariat. Ils sont donc censés être plus combatifs ou au moins autant que les postiers, même si cette vision des choses est en train de changer lentement au milieu des années 1970 dans l'extrême gauche ${ }^{30}$. Celle-ci est par ailleurs relativement coupée de la réalité puisque, selon les Renseignements généraux, l'implantation des gauchistes à la SNCF à cette époque est quasi nulle (la seule implantation certaine est celle de quelques cheminots anarchistes, dont on ne connait pas l'influence exacte mais on peut supposer celle-ci limitée). Leurs appels répétés aux cheminots apparaissent comme des formules incantatoires, à visées performatives, et restent isolés. Fin octobre, l'extrême gauche attend donc une grève générale et déterminée des cheminots.

\section{... Dans les médias}

Le traitement médiatique des cheminots en grève, s'il est légèrement nuancé par la ligne éditoriale de chaque journal, fait apparaitre plusieurs phases, plus ou moins partagées par les quatre quotidiens consultés.

Entre le 24 et le 26 octobre, les cheminots se hissent à la Une de plusieurs quotidiens nationaux. Une grève de certains agents à Montparnasse propulse les cheminots sur le devant de la scène médiatique.

29- Par exemple, on lit dans un supplément à L'Humanité rouge consulté à la BDIC, $\mathrm{n}^{\circ} 25$, en septembre 1969 : « Le démantèlement de la SNCF [sert] à anéantir la combativité des cheminots, fer de lance de la classe ouvrière dans les luttes depuis la Libération. [on veut] anéantir les effectifs de l'armée prolétarienne des cheminots. »

30- Voir Alix Ducamp, «Les travailleurs des services pour l'extrême gauche française des années 1970 : des "cols blancs" à la prolétarisation », Le Mouvement social, $\mathrm{n}^{\circ} 211$ (avril-juin 2005), p. 99-113. 
Par le rubriquage, les cheminots apparaissent donc relativement tôt aux côtés des postiers et se distinguent dans une contestation générale le 25 octobre. Dans Le Parisien, Le Monde et Le Figaro, le «litige avec la direction régionale ${ }^{31}$ de la SNCF, qui pourtant n'est qu'un mouvement circonscrit à la gare de Montparnasse, devient par la mise en page une extension des conflits. La grande attention portée à cet événement fait apparaitre les cheminots comme une menace. Le Figaro, qui se penche sur les raisons de la grève - le refus d'embaucher sept personnes à Montparnasse -, semble faire un parallèle avec le déclenchement, tout aussi local, de la grande grève des PTT, qui est partie d'une brigade du PLM que les responsables refusaient de recevoir ${ }^{32}$. Ce mouvement estil l'étincelle ? Les journaux consultés semblent accréditer cette thèse et marquent donc leur forte attente par rapport à la corporation.

Dans un deuxième temps, entre le 29 octobre et le 5 novembre, cette attente médiatique se renforce, confirmant ainsi l'image d'une corporation combative. Cette période voit en effet l'installation des cheminots à la Une de tous les quotidiens nationaux, alors que le mouvement de grève n'est pas déclenché. Une et gros titres confirment la menace portée par le vocabulaire : "Il ne fait aucun doute que des arrêts de travail pourront avoir lieu à la SNCF au cours de la semaine prochaine. Et probablement faut-il s'attendre à des conflits plus larges ${ }^{33}$. » L'analyse est confirmée par l'éditorial du Monde: «À la SNCF, selon certaines informations, nombre de cheminots envisageraient d'arrêter les trains dans les prochains jours ${ }^{34} »$. C'est l'occasion aussi pour L'Humanité de faire apparaitre à la Une les trois grandes fédérations du combat syndical : les postiers, les mineurs et les cheminots, placés sur un pied d'égalité, à cette différence près que les deux premières corporations sont effectivement en grève et que « des arrêts de travail sont possibles » chez les cheminots ${ }^{35}$. Dans la mise en page, dans les analyses, dans le vocabulaire, les cheminots apparaissent bien comme un espoir ou une menace dans le cadre de la généralisation des conflits. L'analyse semble confirmée par l'étude des descripteurs des journaux télévisés. Sur trente occurrences relatives aux cheminots présentes dans les descripteurs des journaux télévisés durant les mois d'octobre et novembre, 7 se regroupent en trois jours : 31 octobre, 2 et 4 novembre.

31- Le Parisien du 24 octobre.

32- Le Figaro du 24 octobre.

33- Le Figaro du $1^{\text {er }}$ novembre, éditorial de Michel Hamelet, «Pare-feu ».

34- Le Monde du $1^{\mathrm{er}}$ novembre.

35- L'Humanité du $1^{\text {er }}$ novembre. 
À partir du 5 novembre, le mouvement de grève s'engage dans plusieurs régions. Le traitement médiatique change donc au rythme des premières constatations et varie aussi en fonction de la ligne éditoriale des journaux. Dans les premiers jours, les médias entretiennent l'image de menace, le mouvement des cheminots «s'étend $»^{36}$; le 7 novembre, «les perturbations s'aggravent» pour le Parisien et Le Figaro, « les consignes d'arrêt du travail se multiplient » le 8 novembre pour Le Monde, «le mouvement des cheminots se confirme » pour L'Humanité. Mais cette période qui s'achève par une reprise du travail pour le week-end du 11 Novembre, une trêve pour certains, est difficile à appréhender pour les médias. Si les cheminots sont toujours à la Une des quotidiens (excepté Le Parisien où leur présence diminue), ils le sont encore plutôt sous forme de menace ou de promesse. Pourtant apparaissent les premiers doutes alors que s'approche le week-end du 11 Novembre. Le 8 novembre, alors que la SNCF est aux côtés des PTT et de ÉlectricitéGaz de France (EGF) à la Une du Figaro, « on indique la possibilité que les grévistes votent la reprise du travail ${ }^{37} »$. Le même jour, dans Le Monde et L'Humanité, on reconnaît qu' "il n'y a pas jusqu'à présent des perturbations graves ${ }^{38}$. Pour le Parisien, le 9 novembre, « une aube se lève sur le front des grèves ». Une période plus troublée pour l'image des cheminots dont la combativité traditionnelle ne semble pas néanmoins vraiment remise en cause. L'avenir est toujours menaçant ou prometteur chez les cheminots, cette trêve étant destinée à « garder intacte les possibilités de donner une suite à l'action si le gouvernement persiste dans son refus de négocier ${ }^{39}$ pour L'Humanité.

Après le 11 Novembre, lorsqu'un mouvement puissant devient de plus en plus improbable à la SNCF - c'est à ce moment que s'ouvre la troisième phase que nous avons pu dégager - les journaux tendent à occulter les cheminots plutôt qu'à remettre profondément en cause le capital symbolique acquis. Les cheminots quittent ainsi la Une des quotidiens nationaux: une seule Une au Figaro entre le 11 et le 17 novembre (le 15 novembre), pas de présence explicite à la Une du Monde entre le 13 et le 19 et, excepté le 14 novembre, L'Humanité ne fait pas apparaitre les cheminots à sa Une entre le 9 et le 18 novembre. Sur les écrans, trois sujets seulement contenant des images se référant explicitement aux cheminots semblent avoir été diffusés, toutes chaines

36- Le Figaro, 6 et 8 novembre.

37- Le Figaro du 8 novembre.

38- Le Monde du 8 novembre.

39- L'Humanité du 9 novembre. 
confondues. Certes cette période n'est pas une phase d'action mais elle devient vite, à partir du 12 novembre, une période de préparation à l'action et, donc, potentiellement une période de menace dans les journaux. Mais cette fois-ci la menace est moins nette. Elle ne mérite pas les gros titres et les quelques commentaires présents dans les quotidiens semblent plus nuancés qu'au début du mois. Le ton est très neutre dans les entrefilets du Parisien: on annonce de "nouveaux débrayages à la $\mathrm{SNCF} »^{40}$ à partir du 18 dans le cadre d'une grève tournante. Mais l'information ne mérite qu'une dizaine de lignes. Il faut dire que FO, le 12 novembre, vient apaiser la situation. Si les cheminots se déclarent " toujours en état de combativité élevé », la décision prise par FO de ne pas appeler à étendre le mouvement "permettra sans doute de maintenir la circulation des principales lignes ferroviaires $»^{41}$. Le 16 novembre, c'est la décision de la FGAAC qui vient tempérer l'annonce de grève. Le trafic sera donc «quasi normal » pour Le Parisien ${ }^{42}$. Au Figaro, il n'y a quasiment aucun commentaire sur la situation à la SNCF, si ce n'est le 12 novembre où l'on évoque la « surestimation par les syndicats de la combativité de la base $»^{43}$. Le 12 novembre toujours, si des menaces de grève apparaissent à la Une du Monde, on analyse « que le gouvernement mise sur la faible portée du mouvement des cheminots ». Il n'y a guère que L'Humanité pour rappeler la combativité cheminote : le 14 novembre, «les cheminots sont prêts à reprendre l'action $»^{44}$. Toujours est-il que cette période voit une forte réduction de la présence médiatique des cheminots qui n’apparaissent plus comme une menace. Au mieux, ils rentrent dans le rang, reprenant une place commune dans les "points sociaux », les journaux ne faisant qu'annoncer les grèves tournantes du 18 au 22 novembre.

La veillée d'armes qui précède la journée interprofessionnelle d'action du 19 novembre renforce un peu la place des cheminots. Pour L'Humanité du 18 novembre, « ils sont au coude à coude avec les autres travailleurs » et ils ont même droit, puisqu'ils ouvrent, dès le 18, l'action interprofessionnelle, au plus gros titre de la rubrique : « Dès aujourd'hui, les cheminots... ${ }^{45}$ ». L'Humanité est suivie par Le Monde et Le Figaro, le

40- Le Parisien du 15 novembre.

41- Le Parisien du 12 novembre.

42- Le Parisien du 16 novembre.

43- Le Figaro du 12 novembre.

44- L'Humanité du 14 novembre.

45- L'Humanité du 18 novembre. 
19 novembre. C'est le retour des cheminots à la Une. Le Monde annonce que «la grève du 19 novembre devrait surtout entrainer des perturbations dans les transports publics et l'enseignement». Pour le Figaro, c'est « SNCF : perturbations maximales». Les cheminots redeviennent aussi télégéniques : quatre présences dans les journaux télévisés des deux premières chaînes en deux jours, autant que dans la semaine écoulée. Une grande journée d'action interprofessionnelle pourrait-elle se faire sans les cheminots? Sans qu'ils soient parmi les principaux animateurs? Les cheminots apparaissent donc toujours susceptibles de renforcer la contestation.

L'image traditionnelle de combativité semble donc bien présente dans la presse quotidienne. À la veille de chaque action, et même si cela est moins souligné avant la grève du 19, les cheminots sont attendus, parfois avec crainte, parfois avec espoir. Mais quelles sont les réactions des différents acteurs devant le relatif échec du mouvement cheminot ? L'image combative des cheminots est-elle remise en cause ?

\section{Après l'échec du mouvement, les réactions ...}

\section{... De l'extrême gauche}

Les réactions les plus vives à l'échec du mouvement cheminot viennent de l'extrême gauche : c'est aussi parmi ces organisations que les attentes étaient les plus fortes. Ces réactions se font en deux temps. Au début des grèves à la SNCF l'extrême gauche est assez enthousiaste, ne doutant pas du déclenchement d'un grand mouvement cheminot combatif. Par exemple dans la Taupe rouge, le 5 novembre, «les grèves s'étendent. Le meilleur exemple est celui de la SNCF où la grève d'hier à Montparnasse et Invalides s'étend demain à d'autres régions [...] »; le 12 novembre, « la victoire de la grève des PTT est un enjeu pour tous les travailleurs de la fonction publique. [...] Les cheminots l'ont compris qui sont entrés en lutte massivement à Lyon ${ }^{46}$. Puis, au cours du mois de novembre, les militants d'extrême gauche revoient leurs attentes à la baisse : par exemple, dans un article de Lutte ouvrière : «Quant aux cheminots, même militants du PCF, ils ne voient pas très bien ce qui se passe et à quel jeu jouent les fédérations. Ils sont souvent désorientés et certains parlent même de ne pas faire grève la semaine prochaine, surtout parce que le mouvement est un mouvement tournant $t^{47}$. »; ou, dans la Taupe rouge, le 12 novembre : « Le 8 novembre a été un premier pas en avant de l'extension du mouvement au secteur public mais la cessation de la grève des cheminots l'a entravé sérieusement. »

46- Archives de la BDIC, la Taupe rouge, 5 et 12 novembre 1974.

47- Lutte ouvrière, $\mathrm{n}^{\circ}$ 325, daté du 19-25 novembre 1974. 
Même avant la fin des grèves tournantes de la mi-novembre, toute l'extrême gauche, sous des formes diverses et avec une intensité différente selon les organisations, dénonce l'incurie et les manœuvres syndicales, en particulier de la part de la CGT. Ainsi, la Taupe rouge s'interroge le 12 novembre : «l'appel des directions syndicales CGT-CFDT de la SNCF à cesser le mouvement de grève reconductible des cheminots pour d'autres formes d'action n'est-il pas un recul démobilisateur au moment où se prépare la mobilisation des hôpitaux, etc. ? "; le PCR le dit aussi clairement dans un tract ${ }^{48}$, tout en conservant des espoirs vis-àvis des cheminots: "Pourquoi certains responsables syndicaux refusent-ils que les postiers en grève aillent prendre contact avec les cheminots ? [...] ». Dès le milieu du mois de novembre, la LCR dénonce «le sabotage consciemment planifié de la grève à la SNCF ». Elle fait la liste de ces «manœuvres syndicales » : faire croire à la reconductibilité des grèves de 24 heures; pas d'appel national à la grève ; pas de plateforme de revendications communes avec les postiers; un manque d'information et des mensonges sur l'état des grèves à la SNCF pour pousser certains secteurs à la reprise ; les journées interprofessionnelles sans lendemain. Lutte ouvrière, dans un article de son numéro 327 intitulé "La tactique des organisations syndicales ", cherche à en comprendre les raisons. Selon le journal, les syndicats CGT et CFDT ont poussé à l'élargissement de la grève des PTT mais dans une lutte partielle et contrôlée, susceptible de renforcer la réputation de puissance de ces syndicats auprès du gouvernement et du grand public : «Au fond les dirigeants syndicaux ne voulaient mobiliser les cheminots que le temps de la publication de gros titres de journaux, de la lettre de Séguy au gouvernement et de l'ouverture de négociations. » À cette analyse s'ajoute pour les trotskistes la position spécifique de la CGT, très liée au PCF, en particulier à la SNCF, et qui veut donc montrer sa force de mobilisation des travailleurs dans le cadre des négociations PCF-PS pour le Programme commun.

Enfin, l'extrême gauche considère que l'échec à la SNCF est en grande partie à l'origine de l'échec de la grève des postiers. Par exemple, selon Les Cahiers de mai ${ }^{49}$, il n'y a pas eu assez d'unité entre les catégories de grévistes, les revendications de chacune n'étant pas assez connues des autres, et la grève des postiers n'a pas été assez popularisée. Plus clairement, le Rail enchainé fait le lien entre échec à la SNCF et échec des postiers (ce journal anarchiste rapporte l'interview de postiers effectué

48- Archives de la BDIC.

49- Archives de la BDIC, rapport interne, novembre 1974. 
par son homologue des PTT, Le Postier affranchz ${ }^{50}$; le titre de l'article est évocateur: «En nous faisant reprendre le travail le 9 novembre, les directions syndicales ont joué le rôle de fossoyeur de la grève des PTT. »

Cependant la combativité des cheminots n'est pas remise en cause. C'est par exemple le sens du titre de l'article de Rouge s1 $_{\text {"Une réelle }}$ mobilisation, mais... ». Au contraire, les cheminots ont été manipulés par les organisations syndicales dont la « position est claire : il faut tronçonner les luttes, s'adapter à la volonté combative de la base pour mieux la contrôler ", selon le Rail enchain ${ }^{52}$. Ainsi pour Lutte ourrière, "le mécontentement reste profond et les cheminots sont convaincus qu'il faut lutter $»^{53}$. L'extrême gauche affirme que les attentes des cheminots sont déçues alors que ce sont peut-être les siennes qui le sont : c'est un transfert fréquent de la part d'organisations qui prétendent représenter les travailleurs et porter fidèlement leur parole alors qu'il s'agit parfois d'interprétations, plus ou moins stéréotypées.

\section{... Des syndicats}

Les indices de réactions parmi les cheminots ne proviennent que des syndicats (CGT et CFDT essentiellement) et de l'extrême gauche. Ils sont donc empreints de rhétoriques partisanes : écœurement et cartes syndicales déchirées pour les trotskistes et les anarchistes (par exemple, dans le numéro 275 de Rouge, le 22 novembre : «Les manœuvres des directions syndicales - la CFDT n'étant que l'ombre de la CGT omniprésente - sont vivement critiquées : les cheminots jugent et condamnent »), mobilisation et combativité des cheminots qui restent fortes selon la CGT. La CFDT émet des doutes sur la façon dont l'action a été menée et s'interroge sur le moral des cheminots.

Les réactions des syndicats cheminots sont encore plus difficiles à analyser de façon certaine, sauf pour FO qui se félicite de ne pas avoir soutenu la grève des cheminots et d'avoir refusé l'extension de la grève des PTT pour la cantonner à des revendications strictement catégorielles. En effet, d'abord, certains éléments donnent à penser que la CGT a une sorte de mauvaise conscience ou en tout cas se défend des accusations portées contre elle, au-delà même du cercle restreint de l'extrême gauche : dans la Tribune des cheminots de décembre, elle répond à une attaque de la FGAAC selon laquelle le «syndicalisme [de la CGT] a fait faillite» dans ce mouvement: «Pas un seul travailleur, qu'il soit

50- Archives de la BDIC.

51- Rouge, $\mathrm{n}^{\circ} 274$ (15 novembre 1974).

52- Archives de la BDIC. 
agent de conduite ou postier, ne rend responsable la CGT de la nonsatisfaction de ses revendications. Les nombreuses réunions que nous effectuons nous prouvent qu'ils connaissent les véritables responsables : les tenants du pouvoir et ceux du grand capital [...]. » Quant aux attaques de l'extrême gauche, la CGT y répond dans un tract dénonçant, avec la rhétorique habituelle, «l'aventurisme» des gauchistes intellectuels coupés des travailleurs. Dans la Tribune des cheminots de décembre, la CGT au contraire proclame la force de sa fédération : «Sans doute nous dira-t-on [...] que ce n'est pas la majorité des cheminots qui est passée à l'action ces derniers temps. Qu'on y prenne garde! Les quelques 75000 ou 80000 cheminots grévistes dénombrés constituent la partie la plus consciente et la plus combative. » On doit mentionner aussi une sorte de phénomène d'occultation du mouvement à la CGT : curieusement, on l'a dit, il n'y a pas de Tribune des cheminots en novembre 1974 ; de plus, MM. Sgorlon et Lanoue, interrogés sur ce mouvement, ont eu du mal à se le rappeler alors qu'ils étaient cheminots et responsables syndicaux en 1974. On peut aussi préciser qu'il a été impossible de consulter les tracts de la fédération CGT des cheminots (pour des raisons de classement), qui nous auraient sans doute apporté des précisions.

À la CFDT, dans les semaines et les mois qui suivent, la mobilisation des cheminots est l'objet d'interrogations. Si les discussions du secteur action-revendication et du bureau national rappellent la bonne analyse initiale de la CFDT sur la faible combativité du moment des cheminots ${ }^{54}$, elles font apparaitre aussi des interrogations plus profondes sur la corporation, sur le rôle de la fédération CFDT mais aussi sur la place qu'y occupe la fédération CGT. Lors de sa réunion du 28 novembre, le secteur action revendication reconnait « une capacité d'action faible de la CFDT dans le secteur public et nationalisé » et notamment chez les cheminots et à l'EGF, ces deux secteurs étant traditionnellement dominés par la CGT, en constituant ainsi "l'infanterie lors d'actions généralisées $\aleph^{55}$. La faible mobilisation de ces deux secteurs conduit la CFDT à s'interroger sur la capacité de mise en action des secteurs publics et nationalisés par la CGT : est-elle encore aussi forte ? Pourtant, d'après la CFDT, c'est un « brutal coup de frein » dès le 8 novembre des « camarades de la CGT » qui a empêché un bon démarrage du mouvement à la SNCF, comme à l'EGF. Dans le compte rendu qu'il adresse

53- Lutte ouvrière, $\mathrm{n}^{\circ} 324$.

54- Cf. supra.

55- Archives confédérales de la CFDT, Carton $8 \mathrm{H} 400$, activité du secteur actionrevendication, note de réunion du 28 novembre 1974. 
aux fédérations, aux unions départementales ainsi qu'au bureau national et au secrétariat confédéral, le secteur action-revendication semble avoir des difficultés à mesurer l'action des cheminots, notamment pour la journée du 19 novembre : «Dans le secteur nationalisé, une assez bonne participation recouvre cependant une combativité moins grande que la capacité habituelle de ces secteurs. La difficulté la plus importante s'est trouvée chez les cheminots où un accord difficile à obtenir de la CGT pour une grève tournante de quatre jours n'a pas été respecté par cette organisation. Elle a tenté de tout bloquer sur le $19^{56}$. » Le compte rendu du bureau national de la CFDT reprend les interrogations d'un de ses membres sur le « relatif insuccès [de la CFDT] à développer les luttes [...] ailleurs [que dans les PTT] et notamment au niveau de la fonction publique », le même émettant l'idée que n'ayant pu «mobiliser les travailleurs de l'État», la CFDT n'a pas pu « augmenter le rapport de force nécessaire ${ }^{57}$, ce qui apparait comme la première tentative syndicale d'expliquer l'échec aux PTT par la faible mobilisation du secteur public et nationalisé. Mais ici encore, les cheminots ne sont pas désignés directement. Pour tenter de répondre à ces questions, la Fédération CFDT des cheminots construit ainsi une grille d'analyse qui doit être la base du travail en commission et être exploitée lors du conseil fédéral des 10 et 11 décembre. Malheureusement, nous n'avons pas retrouvé le procèsverbal de ce conseil fédéral. Pour autant, elle semble indiquer la volonté de la CFDT de comprendre ce qui s'est passé et manifeste ainsi ses d'interrogations. Cette grille est composée de sept points parmi lesquels des questions sur la perception par les cheminots des actions du 18 au 22 novembre, sur l'impact, chez les cheminots, de la journée du 19, mais aussi sur l'unité d'action CGT-CFDT et sur l'activité des structures $\mathrm{CFDT}^{58}$. Toujours est-il que les conseils fédéraux suivants, notamment celui des 18 et 19 février 1975, font état d'une démobilisation assez importante des cheminots. "Démobilisés par des luttes sans résultats », ils sont « pessimistes » et « moroses ». Toute action au début de l'année 1975, pendant les négociations salariales, apparaît impossible car les «actions de fin d'année avaient épuisé le potentiel de combativité des

56- Ibid., «Pour analyser cette période de lutte», analyse datée 28 novembre 1974, adressée aux fédérations, aux unions départementales, aux unions régionales, au bureau national et au secrétariat confédéral.

57- Archives confédérales de la CFDT, Carton 8H68, compte rendu du bureau national des 5 et 6 décembre 1974.

58- Archives fédérales de la CFDT-Cheminots, Carton 1K27, ordres du jour, rapports et comptes rendus du conseil fédéral de la Fédération des cheminots. 
cheminots $»^{59}$. Pourtant, en octobre 1976, dans le rapport d'activité du $33^{\mathrm{e}}$ congrès de la Fédération des cheminots CFDT, les séquelles ne semblent pas être trop importantes pour celle-ci puisque la « liste des conflits démontre que les cheminots ont su rester réalistes et maintenir leur tradition de lutte », quitte à se distinguer quelquefois des démarches confédérales puisque «bien souvent des actions d'ampleur nationale dans leurs formes ou dans leurs moments se sont situées en marge des choix des choix ou des possibilités déterminés par les travailleurs du rail $[\ldots] \gg^{60}$.

\section{... Des médias}

Si les cheminots semblent éprouver un malaise après le mouvement de novembre et s'ils apparaissent éloignés de leur image traditionnelle de combativité selon la CFDT, peut-on observer ce malaise dans les médias ? Remettent-elles en cause la combativité des cheminots après le mouvement? Cette observation peut se faire en deux temps. Tout d'abord, après la première phase d'action, du 6 au 9 novembre, les médias vont tenter une première analyse de cette grève en demi-teinte. Nous verrons ensuite quels ont été les commentaires des médias après la fin du mouvement.

Après le 11 Novembre, alors que le mouvement des postiers s'enracine mais que le mouvement général peine à démarrer, il est temps pour les médias de revenir sur l'entrée en scène des cheminots. Excepté L'Humanité qui souligne une " combativité » persistante le 12 novembre, les quotidiens nationaux que nous avons pu consulter sont plus sceptiques et relèvent une mobilisation moins forte que prévu. Le 12 novembre, « le gouvernement mise sur la faible portée de la grève des cheminots » pour Le Monde. Ce demi-échec est encore évoqué le 15 novembre quand Le Monde explique «la mobilisation faible à cause de l'augmentation nominale des salaires ». Dans Le Figaro, le 12 novembre, on parle de «la surestimation par les syndicats de la combativité de la base $»^{61}$. Cependant, ces remises en cause, plutôt rares, ne sont jamais directement orientées vers les cheminots, la faible combativité des cheminots est à chaque fois mentionnée pour parler des acteurs de la grève, du gouvernement et des syndicats. Les cheminots sont plutôt considérés comme un terrain de lutte, une arme potentielle parmi d'autres. En effet, dans cette période de généralisation du conflit au secteur public et

59- Ibid., Carton $1 \mathrm{~K} 28$, ordres du jour, rapports et comptes rendus du conseil fédéral de la Fédération des cheminots.

60- Ibid., Carton $1 \mathrm{~K} 9$, congrès de la Fédération des cheminots.

61- Le Figaro du 12 novembre, article de Michel Jacques, "Que veut la CGT ?» 
à quelques entreprises privées, les médias ont tendance à envisager ce mouvement social comme l'affrontement entre les syndicats et le gouvernement dans un cadre de politisation du conflit. Les travailleurs en grève et même les postiers ne sont donc pas considérés comme les acteurs principaux. La combativité traditionnelle des cheminots n'est sans doute pas directement remise en cause. Pour les journaux qui s'opposent à la grève, c'est plutôt l'échec des syndicats que celui des cheminots.

Après le deuxième temps du mouvement des cheminots, alors que la journée interprofessionnelle est diversement appréciée par les journaux, les cheminots disparaissent rapidement de l'espace médiatique. Deux sujets à la télévision évoquent les cheminots le 19 novembre mais on ne trouve rien les jours suivants (jusqu'au 24). Dans la presse, on évoque les cheminots le 20 , pour évaluer leur participation à l'action du 19. Dans L'Humanité, on évoque rapidement la «large participation» des cheminots mais, au Monde, on note "beaucoup moins de perturbations » que lors des précédentes grèves tournantes et on souligne dans un petit reportage : «A Sainte Geneviève-des-Bois, il y a des places assises $^{62}$.» Le Figaro est le plus critique. Dans son éditorial, Xavier Marchetti affirme que « les cheminots sont la preuve que la CGT est en dessous de ses prévisions $\rangle^{63}$. Ensuite, les journaux soulignent la fin des perturbations avec la fin de la grève tournante mais ils ne s'attardent plus sur les cheminots qui disparaissent de l'espace médiatique le 22 novembre. Trois observations peuvent donc être faites : L'Humanité assure un service minimum pour les cheminots ; on en parle mais leur place diminue dans le journal. Les cheminots ne font pas l'objet de longs discours, ni de réflexion particulière et sortent de l'espace médiatique dans un quasi-anonymat dans l'ensemble de la presse. Et enfin, les rares réflexions lues dans Le Figaro et même dans Minute ${ }^{64}$ mettent en cause une stratégie syndicale qui échoue à la SNCF.

La combativité traditionnelle des cheminots ne semble donc pas vraiment remise en cause à court terme. Finalement, il est clair que les cheminots apparaissent plus comme une menace que comme des briseurs de grève. Il faut aussi et surtout tenir compte du fait que le mouvement des cheminots n'est pas l'information principale durant cette période. Même si l'attente des médias quant à l'entrée en grève de

62- Le Monde du 20 novembre.

63- Le Figaro du 20 novembre, éditorial de Xavier Marchetti, «Fausses manœuvres ». 64 - « [...] Les débrayages n’ont pas eu l’ampleur escomptée par les syndicats. Qu’à cela ne tienne, on essaiera un autre secteur [...] », Minute du 20 novembre. 
cette corporation doit être soulignée, il ne faut pas surestimer la place médiatique des cheminots en novembre 1974. Ils apparaissent comme un élément dans un mouvement général. Tous les sujets qui évoquent les cheminots à la télévision sont des " points sociaux » et, la plupart du temps, les cheminots n'apparaissent jamais seuls dans la presse, à la Une comme en page intérieure. Les articles qui sont consacrés au mouvement des cheminots ne sont jamais très longs et ne prennent pas le temps d'expliquer en profondeur ce qui se passe dans la corporation. Les journaux rappellent parfois les revendications, annoncent les journées d'actions et surtout les prévisions de trafic avec une précision assez éloquente : on s'intéresse aux conséquences de la grève des cheminots mais ni à ses causes ni aux cheminots eux-mêmes. Il convient donc sans doute de ne pas surestimer le rôle des cheminots dans le conflit luimême et la place qu'ils occupent dans l'espace médiatique. De ce point de vue, les postiers apparaissent sans égal pour la combativité en cet automne 1974. S'il faut trouver une remise en cause médiatique de la traditionnelle combativité des cheminots, elle est sans doute davantage dans la comparaison avec les postiers que dans l'analyse de la corporation elle-même.

On voit bien que novembre 1974 est un mouvement de grève très controversé pour les cheminots et qui pose de nombreuses questions, auxquelles nous n'avons pas toujours pu répondre, faute de sources.

On peut d'abord se demander si cet épisode a eu des répercussions sur un futur mouvement. Ce n'est pas le cas selon les RG qui restent sur le qui-vive d'après leurs rapports des années suivantes. En revanche, on peut imaginer que cet échec en 1974 a pu engendrer une baisse de confiance envers les syndicats ou une baisse de moral parmi les cheminots (c'est la thèse de l'extrême gauche), d'autant que l'aprèsMai 68 et notamment l'année 1974 connaissent un contexte de fortes luttes sociales. Ce facteur a d'ailleurs pu être une circonstance aggravante pour la perception de la combativité cheminote. Mais, finalement, il est tout aussi probable que cet échec soit relativement passé inaperçu, en tout cas pour le grand public, comme le prouve la quasi-absence de commentaires de la presse à la fin du mouvement. Les critiques de l'extrême gauche, les questionnements internes aux syndicats ne bénéficient évidemment pas d'une large diffusion. Même pour l'extrême gauche il ne s'agit pas d'un échec des cheminots mais de la faillite des grands syndicats engagés dans une collaboration avec l'État et le patronat. 
Cet échec qui ne correspond pas au mythe cheminot est sans doute à replacer dans le début du reflux du mouvement ouvrier et de ses organisations politiques et syndicales dans les années 1970 et 1980. Il y a par la suite de grands mouvements chez les cheminots : dans les années 1980, en 1995. Si novembre 1974 est une parenthèse dans le mythe de la combativité cheminote, on ne peut pas dire avec certitude que cet épisode ait ébranlé la réputation, la perception de la combativité des cheminots dans la société. Tout au plus novembre 74 a-t-il pu placer les postiers au centre de l'action revendicative, si l'on prend les médias comme sources essentielles des représentations dans la société. 